the vector by a pathogen, providing a theoretical framework for its impact on human health and disease. These and related findings demonstrate how relevant ecology and vector biology are in protecting human and animal health, especially for emerging zoonotic and vector-borne diseases.

\section{Acknowledgments}

This work was supported in part by NIH/ NIAID R01 AI44102 and R21 AI080911.

Address correspondence to: J. Stephen Dumler, Division of Medical Microbiology, Department of Pathology, 720 Rutland Avenue, Ross Building Room 624, Baltimore, Maryland 21205, USA. Phone: 410.955.8654; Fax: 443.287.3665; E-mail: sdumler@jhmi.edu.

1. Woolhouse ME, Gowtage-Sequeria S. Host range and emerging and reemerging pathogens. Emerg Infect Dis. 2005;11(12):1842-1847.

2. Jones KE, et al. Global trends in emerging infectious diseases. Nature. 2008;451(7181):990-993.

3. Walker DH, Paddock CD, Dumler JS. Emerging and re-emerging tick-transmitted rickettsial and ehrlichial infections. Med Clin North Am. 2008;92(6):1345-1361

4. Neelakanta G, Sultana H, Fish D, Anderson JF, Fikrig E. Anaplasma phagocytophilum induces Ixodes scapularis ticks to express an antifreeze glycoprotein gene that enhances their survival in the cold. J Clin Invest. 2010;120(9):3179-3190.

5. McNabb SJ, et al. Summary of notifiable diseases--
United States, 2006. MMWR Morb Mortal Wkly Rep. 2008;55(53):1-92.

6. Paddock CD, Holman RC, Krebs JW, Childs JE. Assessing the magnitude of fatal Rocky Mountain spotted fever in the United States: comparison of two national data sources. Am J Trop Med Hyg. 2002;67(4):349-354.

7. Paddock CD, et al. Hidden mortality attributable to Rocky Mountain spotted fever: immunohistochemical detection of fatal, serologically unconfirmed disease. J Infect Dis. 1999; 179(6):1469-1476.

8. Angerami RN, et al. Clusters of Brazilian spotted fever in Sao Paulo State, southeastern Brazil. A review of official reports and the scientific literature. Clin Microbiol Infect. 2009;15(suppl 2):202-204.

9. Openshaw JJ, et al. Rocky Mountain spotted fever in the United States, 2000-2007: Interpreting contemporary increases in incidence. Am J Trop Med Hyg. 2010;83(1):174-182.

10. Dumler JS, Madigan JE, Pusterla N, Bakken JS Ehrlichioses in humans: epidemiology, clinical presentation, diagnosis, and treatment. Clin Infect Dis. 2007;45(suppl 1):S45-S51.

11. Carlyon JA, Fikrig E. Mechanisms of evasion of neutrophil killing by Anaplasma phagocytophilum. Curr Opin Hematol. 2006;13(1):28-33.

12. Garcia-Garcia JC, Barat NC, Trembley SJ, Dumler JS. Epigenetic silencing of host cell defense genes enhances intracellular survival of the rickettsial pathogen Anaplasma phagocytophilum. PLoS Pathog. 2009;5(6):e1000488.

13. Garcia-Garcia JC, Rennoll-Bankert KE, Pelly S, Milstone AM, Dumler JS. Silencing of host cell CYBB gene expression by the nuclear effector AnkA of the intracellular pathogen Anaplasma phagocytophilum. Infect Immun. 2009;77(6):2385-2391

14. Borjesson DL, Kobayashi SD, Whitney AR, Voyich JM, Argue CM, Deleo FR. Insights into pathogen immune evasion mechanisms: Anaplasma phagocytophilum fails to induce an apoptosis differen- tiation program in human neutrophils. J Immunol. 2005;174(10):6364-6372.

15. Teglas MB, Foley J. Differences in the transmissibility of two Anaplasma phagocytophilum strains by the North American tick vector species, Ixodes pacificus and Ixodes scapularis (Acari: Ixodidae). Exp Appl Acarol. 2006;38(1):47-58.

16. Brinza L, et al. Systemic analysis of the symbiotic function of Buchnera aphidicola, the primary endosymbiont of the pea aphid Acyrthosiphon pisum. Comptes Rendus Biologies. 2009;332(11):1034-1049.

17. Pais R, Lohs C, Wu Y, Wang J, Aksoy S. The obligate mutualist Wigglesworthia glossinidia influences reproduction, digestion, and immunity processes of its host, the tsetse fly. Appl Environ Microbiol. 2008;74(19):5965-5974.

18. Saridaki A, Bourtzis K. Wolbachia: more than just a bug in insects genitals. Curr Opin Microbiol. 2010;13(1):67-72.

19. Houhamdi L, Fournier PE, Fang R, Lepidi H, Raoult D. An experimental model of human body louse infection with Rickettsia prowazekii. J Infect Dis. 2002;186(11):1639-1646.

20. Niebylski ML, Peacock MG, Schwan TG. Lethal effect of Rickettsia rickettsii on its tick vector (Dermacentor andersoni). Appl Environ Microbiol. 1999;65(2):773-778.

21. Scorpio DG, Akkoyunlu M, Fikrig E, Dumler JS. CXCR2 blockade influences Anaplasma phagocytophilum propagation but not histopathology in the mouse model of human granulocytic anaplasmosis. Clin Diagn Lab Immunol. 2004;11(5):963-968.

22. Choi KS, Scorpio DG, Dumler JS. Anaplasma phagocytophilum ligation to toll-like receptor (TLR) 2, but not to TLR4, activates macrophages for nuclear factor-kappa B nuclear translocation. J Infect Dis. 2004;189(10):1921-1925.

23. Thomas V, Samanta S, Wu C, Berliner N, Fikrig E. Anaplasma phagocytophilum modulates gp91phox gene expression through altered interferon regulatory factor 1 and PU.1 levels and binding of CCAAT displacement protein. Infect Immun. 2005;73(1):208-218.

\title{
Too much PABP, too little translation
}

\author{
Hemant K. Kini, ${ }^{1}$ Melanie R. Vishnu, ${ }^{1}$ and Stephen A. Liebhaber ${ }^{1,2}$
}

${ }^{1}$ Department of Genetics and ${ }^{2}$ Department of Medicine, University of Pennsylvania School of Medicine, Philadelphia, Pennsylvania, USA.

\begin{abstract}
Posttranscriptional regulation is of critical importance during mammalian spermiogenesis. A set of mRNAs that encode proteins critical to normal sperm formation are synthesized early in the process of male germ cell differentiation and are stored in a repressed state. These mRNAs are subsequently translationally activated during the process of spermatid elongation and maturation. Of note, the translationally repressed mRNAs contain long poly(A) tails that are dramatically shortened during the translational activation process. Understanding the mechanisms that underlie this process of mRNA storage and subsequent translational activation has been a long-standing goal. The relationship of the poly(A) tail to translational control is intimately related to the functions of the cognate poly(A)-binding proteins (PABPs). In this issue of the JCI, Yanagiya and colleagues use a set of knockout mice to demonstrate a novel functional role for a particular modulator of PABP function, PABP-interacting protein 2a (PAIP2A), in the normal terminal differentiation of male germ cells.
\end{abstract}

Conflict of interest: The authors have declared that no conflict of interest exists.

Translational control is critical to germline formation

Modulation of mRNA translation is an effective tool to regulate gene expression in the absence of new mRNA synthesis. In part, translation is postulated to be affected by the $5^{\prime}$ methyl-7-guanosine $\left(\mathrm{m}^{7} \mathrm{G}\right)$ cap and $3^{\prime}$ poly(A) tail, two distinguishing features of a typical mature mRNA. Binding of eukaryotic initiation factor $4 \mathrm{E}$ (eIF4E) to the $5^{\prime}$ cap and poly(A)- binding protein (PABP) to the poly(A) tail precedes translational initiation. According to current models, the initiation factor eIF4G can bind simultaneously to both eIF4E and PABP, thus bridging the ends of the transcript. These interactions are proposed to circularize the mRNA, forming a postulated "closed loop" mRNA structure (1). This structure protects the mRNA termini from nuclease attack and enhances translational activity (2). Thus, poly(A) tail length and the poly(A) packaging protein PABP are considered to be critical determinants of both the stability and 
A

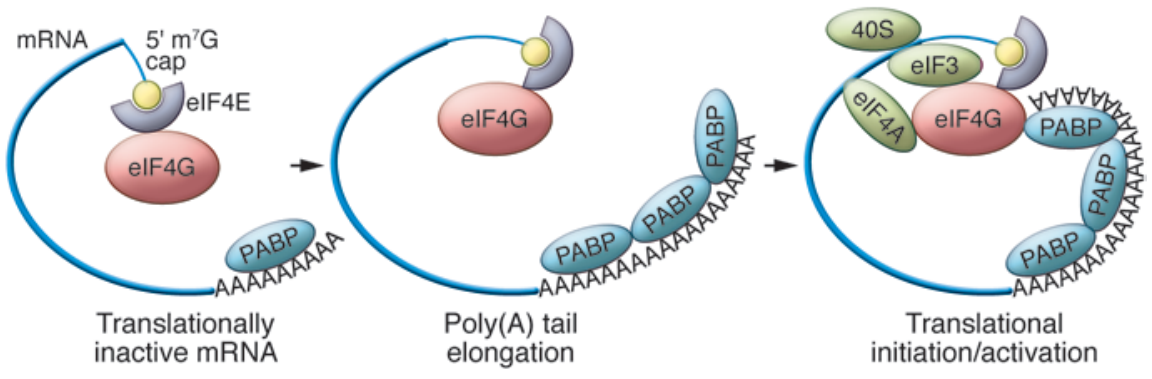

Figure 1

Canonical pathway of developmentally regulated translational activation via poly $(A)$ elongation. In this model, which has been delineated in detail during oocyte maturation and in the early embryo in a number of species $(3,18)$, maternal mRNAs are stored in a repressed state with very short poly $(A)$ tails $(A)$. Their translational activation is mediated by poly $(A)$ tail elongation (cytoplasmic polyadenylation), with subsequent PABP binding (B). These interactions result in a proposed mRNA closed loop formation and translational activation (C).

translational activity of an mRNA. As a general rule, it has been observed that elongation of the poly $(\mathrm{A})$ tail enhances translation and that PABP constitutes a trans-activator critical to this process (Figure 1).

In a variety of well-documented models of early development, it has been observed that subsets of maternal mRNAs are stored in a translationally inert form in the cytoplasm (3). This state of suspended animation is usually dependent on a preceding step where the poly(A) tails of these mRNAs are shortened to such a degree that PABP can presumably no longer bind in levels sufficient to support translation (4). The subsequent translational activation of these stored mRNAs during meiotic maturation or early embryogenesis has been attributed to poly(A) elongation, a process referred to as cytoplasmic polyadenylation $(3,5)$.

Thus, what is so striking and singular about translational control in mammalian spermiogenesis is the apparent reversal of this pattern. Spermiogenesis involves a progression in cell differentiation from spermatogonia to primary (meiotic) spermatocytes, to round and elongating spermatids, and finally to mature sperm (6). Late in this process, a set of mRNAs that have been synthesized and stored in a polyadenylated and translationally repressed state are translationally activated. Remarkably, this translational activation tracks with poly(A) shortening. This process appears to control the expression of the genes encoding male germ cellspecific proteins such as protamines (Prms) and transition proteins (Tps). These polyadenylated mRNAs are bound by the translational repressors MSY2 and MSY4 $(7,8)$. The resulting messenger ribonucleoprotein particle (mRNP) is translationally quiescent and appears to be resistant to mRNA decay. During this phase of translational repression, poly(A) tails on these mRNAs are approximately 160 bases long (Figure 2A). Once these mRNAs are shifted to translationally active

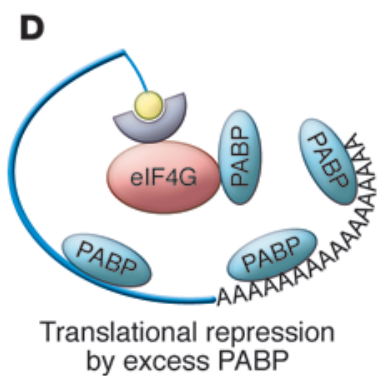

\section{Figure 2}

polysomal fractions in elongating spermatids, the poly(A) tails are reduced to approximately 30 bases (9) (Figure 2, B and C). Currently it is unknown whether this poly(A) tail shortening is a prerequisite for translational activation, or if it is merely a secondary by-product of the translation process. Due to the inherent challenges in reproducing the translational environment of late spermiogenesis in vitro, this mechanism of translational activation and its temporal linkage to poly(A) shortening have been difficult to study and have remained open to much speculation. In this issue of the JCI, Yanagiya and colleagues reveal a novel role for PABP-interacting protein 2a (PAIP2A) in maintaining effective spermiogenesis (10). This protein appears to play an essential role in the process of translational activation of mRNAs encoding testis-specific proteins.

\section{Knockout of Paip2a inhibits translational activation and blocks effective spermiogenesis in mouse}

It is known from prior studies that the impact of PABP on translation is modulated

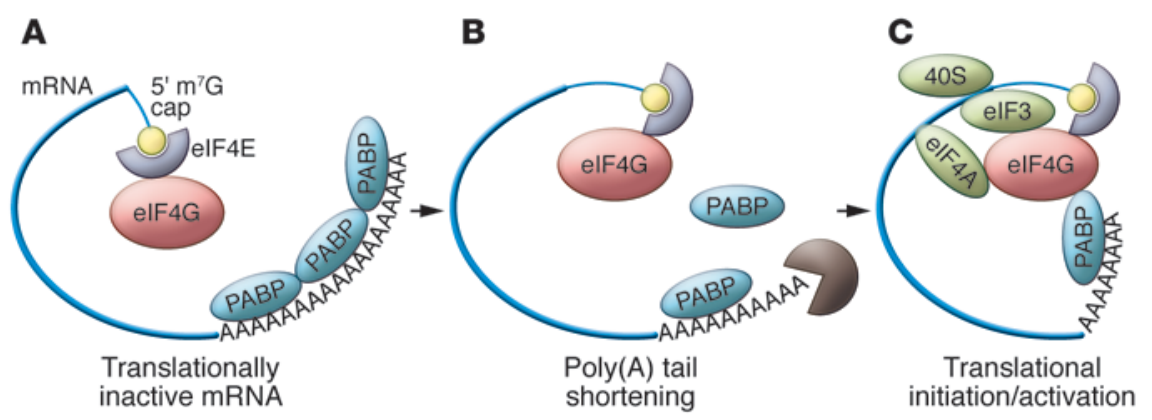

Male germ cell-specific translational initiation/activation. mRNAs generated early in spermatogenesis are stored with long poly $(A)$ tails $(\mathbf{A})$. Their subsequent activation during the terminal phases of sperm differentiation is associated with shortening of the poly $(A)$ tail $(B)$ and translational activation (C). The underlying mechanisms of activation remain unclear, and the question of whether the poly $(A)$ shortening plays an active role in this process remains unanswered. The study by Yanagiya et al. (10) demonstrates that this pathway is in some way critically dependent on the presence of Paip2a. They propose that the knockout of Paip2a results in a pathologic retention of high levels of PABP in the late stages of spermatogenesis (D). In Paip2a-KO mice (10), the abundant PABP may interfere with normal interactions of poly(A)-bound PABP with the elF4G by direct competition and/or by nonspecific coating of the mRNA, with consequent alterations in overall mRNA structure. The role of Paip2a in controlling PABP levels and/or its more specific role(s) in the translational activation pathway remain undefined. 
by two PABP-interacting proteins, PAIP1 and PAIP2 (11). PAIP1, in association with the RNA helicase eIF4A and the 40S ribosomebinding factor eIF3, stabilizes the interaction of PABP with eIF4G and thereby stimulates translation $(12,13)$. In contrast, the two PAIP2 isoforms, PAIP2A and PAIP2B, act to repress translation by reducing $\mathrm{PABP}$ affinity for the poly $(\mathrm{A})$ tail and blocking the interaction of PABP with eIF4G $(14,15)$. The roles of these PAIP2 isoforms in translational control have not been previously explored in vivo to any significant degree.

In this issue of the JCI, Yanagiya et al. (10) approach this problem by using a gene knockout approach. They report that systemic homozygous interruption of the Paip2a locus alone, or in combination with interruption of the Paip $2 b$ locus, results in infertility in male mice, with a corresponding morphologic defect in late spermatogenesis. Of note, the PAIP2A and PAIP2B isoforms appear to be functionally quite similar in that they have equal affinities for PABP and appear to regulate translation in the same manner (15). It is remarkable, therefore, that deletion of Paip $2 b$ does not have the same detrimental effect on spermiogenesis as seen upon Paip2a deletion. This finding suggests that PAIP2A has a unique and predominant role in spermatogenesis. The Paip2a knockout results in a drastic decrease in the levels of expression of Prm1, Tp1, and Tp2 proteins relative to those in wild-type mice. The expression of these proteins, which are critical to sperm maturation, is normally under translational control (described above). Yanagiya et al. demonstrate that under normal circumstances, levels of Pabp decrease, while those of Paip2a increase during the transition from round spermatids to elongating spermatids, corresponding with the translational activation in the elongating spermatids (10). In the Paip2a-KO mouse, Pabp levels were abnormally sustained at high levels. Based on their studies, the authors propose a model whereby loss of PAIP2A results in a reciprocal increase in PABP levels in elongating spermatids by disrupting a putative PAIP2A-mediated PABP degradation pathway (10). The abnormally high levels of PABP are proposed to block the pathway of translational activation of critical mRNAs, with consequent defects in sperm development.

\section{PAIP2A: both a repressor and an activator of translation}

This study by Yanagiya et al. (10), while demonstrating the vital role of PAIP2A in spermiogenesis, also raises several questions pertaining to its specific role in translational regulation. Of particular importance is the question of whether (and how) PAIP2A regulates levels of PABP. Yanagiya and colleagues observed a moderate increase in Pabp protein levels in both Paip2a single knockouts and mice lacking both Paip2a and Paip2b. These data suggest that there is a reciprocal relationship between PAIP2A and PABP. While it has been previously established that Paips regulate the activity of $\operatorname{PABP}(12,16)$, the mechanism by which PAIP2 modulates the levels of PABP expression has yet to be identified. Although PABP has been show to autoregulate the translation of its own mRNA by binding to an adenine-rich region in its $5^{\prime}$ UTR (17), this mechanism does not appear to counteract the overexpression of Pabp that Yanagiya and colleagues observed in the Paip2a-KO mice (10). Thus, the cause of Pabp upregulation in these animals remains unclear.

Given that the Paip2a knockout results in overexpression of Pabp, it was pertinent to explore how this overexpression impacts translation (10). Using an in vitro assay, Yanagiya and colleagues demonstrate that high levels of recombinant PABP can inhibit translation and that the addition of PAIP2A to the system can reverse this effect (10). Thus, these findings are intriguing because (a) PABP, a proposed translation initiation factor, appears to inhibit translation when present at high levels; and (b) PAIP2A, a known translational repressor, functions to enhance translation by neutralizing the excess PABP. These findings indicate that, at least in this in vitro translation system, there is an optimal level for PABP. It remains to be determined whether the concentrations of recombinant PABP or PAIP2A used in these in vitro assays are physiologically relevant and/or if the fold change in PABP concentration needed to achieve translational inhibition in the in vitro system is representative of the alterations in Pabp levels seen in the Paip2a-KO mice.

How does the loss of Paip2a block translation? Yanagiya and colleagues propose that the principal effect of the Paip2a knockout is the generation of excess Pabp in terminally differentiating spermatocytes (10). This excess of Pabp may have two detrimental effects on mRNA translation. The first is that at high concentrations, it may bind throughout the mRNA in a nonspecific fashion, thus altering overall mRNP structure and function. In a more direct manner, the excess free PABP may compete with poly(A)-bound PABP for eIF4G interactions (Figure 2D). This would be predicted to disrupt mRNA circularization and suppress translation. Alternatively, it is formally possible that PAIP2A may play a direct role in the translational activation of mRNAs in the elongating spermatocyte, and the in vivo defect in the Paip2a-KO mouse may be a direct reflection of the loss of Paip2a actions per se rather than having anything to do with observed alterations in Pabp levels (10). For example, the role of PAIP2A as a PABP-interacting protein may be critical in establishing the native structure and/or organization of the repeating $\mathrm{PABP} /$ poly $(\mathrm{A})$ RNP structures. This would be consistent with the subtle changes in the packaging of the poly (A) tail as revealed in the study by Yanagiya et al. (10). Such changes might have a downstream impact on cap functions and the assembly of the translational apparatus. It is of interest to note that the knockout of Paip $2 a$ and consequent loss of translational activation did not appear to be accompanied by a substantial alteration in poly(A) tail size of the male germline-specific mRNAs in the testis when compared with wild-type controls (10). Thus, while Yanagiya and colleagues present strong evidence for a role for PAIP2A in sperm development and function, the mechanisms involved, such as its potential effect on translational biochemistry and relationship to the MSY2/ MSY4 pathway of translational repression, remain to be more fully explored.

\section{Acknowledgments}

M.R. Vishnu was supported by NIH grant T32-DK07780. S.A. Liebhaber is the recipient of a MERIT Award from the NIH (R37-HL 65449).

Address correspondence to: Stephen A. Liebhaber, Departments of Genetics and Medicine, University of Pennsylvania School of Medicine, Room 560A CRB, 415 Curie Blvd, Philadelphia, Pennsylvania 19104, USA. Phone: 215.898.7834; Fax: 215.573.5157; E-mail: liebhabe@mail.med.upenn.edu.

1. Wells SE, Hillner PE, Vale RD, Sachs AB. Circularization of mRNA by eukaryotic translation initiation factors. Mol Cell. 1998;2(1):135-140.

2. Preiss T, Hentze MW. Starting the protein synthesis machine: eukaryotic translation initiation. Bioessays. 2003;25(12):1201-1211

3. Richter JD. Cytoplasmic polyadenylation in development and beyond. Microbiol Mol Biol Rev. 1999; 63(2):446-456.

4. Kim JH, Richter JD. Opposing polymerase-deadenylase activities regulate cytoplasmic polyadenylation. Mol Cell. 2006;24(2):173-183.

5. Sheets MD, Fox CA, Hunt T, Vande Woude G, 
Wickens M. The 3 '-untranslated regions of c-mos and cyclin mRNAs stimulate translation by regulating cytoplasmic polyadenylation. Genes Dev. 1994;8(8):926-938.

6. Mays-Hoopes LL, Bolen J, Riggs AD, Singer-Sam J. Preparation of spermatogonia, spermatocytes, and round spermatids for analysis of gene expression using fluorescence-activated cell sorting. Biol Reprod. 1995;53(5):1003-1011.

7. Giorgini F, Davies HG, Braun RE. Translational repression by MSY4 inhibits spermatid differentiation in mice. Development. 2002;129(15):3669-3679.

8. Yang J, Morales CR, Medvedev S, Schultz RM, Hecht NB. In the absence of the mouse DNA/RNA-binding protein MSY2, messenger RNA instability leads to spermatogenic arrest. Biol Reprod. 2007;76(1):48-54.

9. Kleene KC. Poly(A) shortening accompanies the activation of translation of five mRNAs dur- ing spermiogenesis in the mouse. Development. 1989;106(2):367-373.

10. Yanagiya A, Delbes G, Svitkin YV, Robaire B, Sonenberg N. The poly(A)-binding protein partner Paip2a controls translation during late spermiogenesis in mice. J Clin Invest. 2010;120(9):3389-3400.

11. Derry MC, Yanagiya A, Martineau Y, Sonenberg N. Regulation of poly(A)-binding protein through PABP-interacting proteins. Cold Spring Harb Symp Ouant Biol. 2006;71:537-543.

12. Craig AW, Haghighat A, Yu AT, Sonenberg N. Interaction of polyadenylate-binding protein with the eIF4G homologue PAIP enhances translation. Nature. 1998;392(6675):520-523.

13. Martineau $\mathrm{Y}$, et al. Poly(A)-binding protein-interacting protein 1 binds to eukaryotic translation initiation factor 3 to stimulate translation. Mol Cell Biol. 2008;28(21):6658-6667.
14. Khaleghpour K, et al. Translational repression by a novel partner of human poly(A) binding protein, Paip2. Mol Cell. 2001;7(1):205-216.

15. Berlanga JJ, Baass A, Sonenberg N. Regulation of poly(A) binding protein function in translation: Characterization of the Paip2 homolog, Paip2B. RNA. 2006;12(8):1556-1568.

16. Yoshida M, et al. Poly(A) binding protein (PABP) homeostasis is mediated by the stability of its inhibitor, Paip2. EMBO J. 2006;25(9):1934-1944.

17. Wu J, Bag J. Negative control of the poly(A)-binding protein mRNA translation is mediated by the adenine-rich region of its $5^{\prime}$-untranslated region. J Biol Chem. 1998;273(51):34535-34542.

18. Radford HE, Meijer HA, de Moor CH. Translational control by cytoplasmic polyadenylation in Xenopus oocytes. Biochim Biophys Acta. 2008;1779(4):217-229.

\title{
Transgenic animals may help resolve a sticky situation in cystic fibrosis
}

\author{
Jonathan $\mathrm{H}$. Widdicombe
}

Department of Physiology and Membrane Biology, University of California Davis, Davis, California, USA.

\begin{abstract}
Cystic fibrosis (CF) is caused by defects in the CFTR, a cAMP-activated $\mathrm{Cl}^{-}$ channel of epithelia. The resulting reduction in epithelial fluid transport creates abnormally viscous secretions from airway mucous glands that may be a major factor in CF pathology. Mouse airways have few mucous glands, and the mouse model of $\mathrm{CF}$ exhibits no significant airway disease. Pigs and ferrets, however, have approximately the same number of airway mucous glands as humans. In this issue of the JCI, three independent research groups conclude that changes in airway mucous gland function in CFTR-deficient animals of these species resemble the changes seen in human CF. It is expected, therefore, that these animals will develop lung disease similar to human CF and prove to be valuable models on which to test potential therapies.
\end{abstract}

The body's main defense against large inhaled particles is the "mucociliary clearance system." This system traps particles in a blanket of mucus and then moves the mucus and ensnared particles out of the airways using cilia in the apical membrane of the surface epithelium (1). In the larger airways, the great majority of mucus comes from submucosal glands (2), and mucous secretions from these glands are abnormally viscous in individuals with cystic fibrosis (CF) (3). It is believed that this causes the mucus to be poorly cleared by cilia and it accumulates and provides a home for inhaled microorganisms. An inflammatory cascade ensues that clogs the airways with mucous secretions, bacteria, leukocytes,

Conflict of interest: The author has declared that no conflict of interest exists.

Citation for this article: J Clin Invest. 2010; 120(9):3093-3096. doi:10.1172/JCI44235. and plasma transudate. By restoring normal viscosity to $\mathrm{CF}$ airway mucous gland secretions, it is to be hoped that much of this pathology can be prevented.

Mouse airways contain few mucous glands, and the mouse model of CF shows little airway pathology (4). However, more direct analyses of the role of mucous glands in the airway pathology of CF are now possible, given the recent development of CFTR-deficient pigs and ferrets $(5,6)$; both species have good numbers of airway glands. In this issue of the $J C I$, three independent research groups report on their efforts to understand airway gland function in these new animal models of CF (7-9).

\section{Airway mucous gland secretion in CF}

Water accounts for approximately $98 \%$ of airway gland mucous secretions, and water flow into the gland lumen is driven by local osmotic gradients generated by active $\mathrm{Cl}^{-}$secretion. This $\mathrm{Cl}^{-}$secretion requires simultaneous activity of $\mathrm{Cl}^{-}$channels (either cAMP or $\mathrm{Ca}^{2+}$ activated) in the apical membrane and $\mathrm{K}^{+}$channels in the basolateral membrane. In CF secretory responses of airway glands to agents that elevate cAMP are almost completely lost, as the only cAMP-activated $\mathrm{Cl}^{-}$channel in the apical membrane is CFTR (10). Secretory responses to agents, such as substance $\mathrm{P}$, that moderately elevate intracellular $\mathrm{Ca}^{2+}$ concentration $\left(\left[\mathrm{Ca}^{2+}\right]_{i}\right)$ are also quite markedly reduced in CF (11). This is because $\left[\mathrm{Ca}^{2+}\right]_{i}$ is not elevated sufficiently to have much effect on $\mathrm{Ca}^{2+}$-activated $\mathrm{Cl}^{-}$ channels (CaCCs) in the apical membrane, but $\mathrm{Ca}^{2+}$-dependent basolateral $\mathrm{K}^{+}$channels are opened, thereby hyperpolarizing the apical membrane and increasing the driving force for $\mathrm{Cl}^{-}$exit through constitutively open CFTR. By contrast, responses to cholinergic agents are less affected in CF, because these agents have a larger effect on $\left[\mathrm{Ca}^{2+}\right]_{i}$ and cause substantial activation of $\mathrm{CaCCs}(10-12)$. Finally, in nonCF airway glands, subthreshold doses of cAMP-elevating and $\mathrm{Ca}^{2+}$-elevating agents show synergy; neither alone stimulates gland fluid secretion, but they do so in combination, because one opens CFTR and the other opens basolateral $\mathrm{Ca}^{2+}$-activated $\mathrm{K}^{+}$channels $(10-12)$. This synergy is lost in CF (10). 